\title{
Studies on Nitrogen Substitution through Organics and Foliar Application of Water Soluble Fertilizers on Chemical Parameters and Micro Nutrient Uptake by Chilli (Capsicum annuum L.) In a Vertisol
}

\author{
T. Thulasiramireddy*, B.I. Bidari, P.L. Patil and M.V. Manjunatha \\ Department of Soil Science and Agricultural Chemistry, \\ University of Agricultural Sciences, Dharwad - 580 005, Karnataka, India \\ *Corresponding author
}

\begin{abstract}
A B S T R A C T
A field experiment was conducted during kharif 2013-14 and 2014-15 on Byadgi chilli by substituting fertilizer nitrogen with different sources of organic manures along with foliar application of water soluble fertilizers to study the micro nutrient uptake by chilli. The

Keywords

Capsaicin,

Oleoresin and

Micronutrients

Article Info

Accepted:

20 November 2018

Available Online:

10 December 2018 experiment consists of four main treatments as organics, $\mathrm{M}_{1}$ - Recommended Package of Practices (RPP)- $\left\{\right.$ Recommended Dose of Fertilizers (RDF) $\left.+25 \mathrm{t} \mathrm{ha}^{-1} \mathrm{FYM}\right\}, \mathrm{M}_{2}-50$ per cent $\mathrm{N}$ through $\mathrm{FYM}+50$ per cent inorganic $\mathrm{N}, \mathrm{M}_{3^{-}} 50$ per cent $\mathrm{N}$ through $\mathrm{VC}+50$ per cent inorganic $\mathrm{N}, \mathrm{M}_{4^{-}} 50$ per cent $\mathrm{N}$ through $\mathrm{FYM}$ and $\mathrm{VC}+50$ per cent inorganic $\mathrm{N}$ and four sub treatments as water soluble fertilizers, $\mathrm{S}_{1}-\mathrm{KNO}_{3} @ 1$ per cent, $\mathrm{S}_{2}-\mathrm{K}_{2} \mathrm{SO}_{4} @ 1$ per cent, $\mathrm{S}_{3}$ - 19:19:19 @ 1 per cent, $\mathrm{S}_{4}-\mathrm{KNO}_{3}+\mathrm{K}_{2} \mathrm{SO}_{4}+$ 19:19:19 each @ 1 per cent along with one control (RPP + water spray) with three replications. The design adopted was split plot. Results revealed that combined foliar application of $\mathrm{KNO}_{3}+\mathrm{K}_{2} \mathrm{SO}_{4}+19: 19: 19$ each @ one per cent recorded highest capsaicin ( 0.14 per cent) and oleoresin ( 16.35 per cent) content and substitution of 50 per cent nitrogen through vermicompost +50 per cent nitrogen through inorganics noticed higher iron uptake $\left(852.9,921.6\right.$ and $1010.9 \mathrm{~g} \mathrm{ha}^{-1}$ at 75,105 and 140 DAT respectively), zinc uptake $\left(100.1,104.9\right.$ and $147 \mathrm{~g} \mathrm{ha}^{-1}$ at 75,105 and 140 DAT respectively), manganese uptake $\left(274.7,383\right.$ and $408.9 \mathrm{~g} \mathrm{ha}^{-1}$ at 75,105 and 140 DAT respectively) and copper uptake $\left(48.68,49.56\right.$ and $51.35 \mathrm{~g} \mathrm{ha}^{-1}$ at 75,105 and 140 DAT respectively).
\end{abstract}

\section{Introduction}

Chilli (Capsicum annuиm L.) belongs to the family solanaceae and has a tremendous economic importance. It is also very popular among the people for its medicinal value because the extract of chili is used in different pharmaceutical products. The pungency in chilli is due to the presence of a volatile alkaloid 'Oleoresin capsaicin', a major alkaloid among capsaicinoids produced only in Capsicum fruits (Prasad, 2006). Chilies are highly nutritive and contain a high proportion of digestible proteins, carbohydrates, minerals and vitamins. The sweet pepper commonly known as chili is the world's third most 
important vegetable after tomato and potato (FAO, 2007). Chili is an important vegetable crop used as condiment, salad and food spice. $\mathrm{K}$ is not a constituent of any organic molecule or plant structure, it is involved in numerous biochemical and physiological processes vital to plant growth, yield, quality, and stress tolerance. In addition to stomatal regulation of transpiration and photosynthesis, potassium is also involved in photophosphorylation, enhance enzyme activation, turgor maintenance, and stress tolerance. Adequate K nutrition has also been associated with increased yields, fruit size, increased soluble solids and ascorbic acid concentrations, improved fruit color, increased shelf life, and shipping quality of many horticultural crops (Lester et al., 2010).

Uptake of nutrients from soil by crop plants is directly correlated with the enhanced yield, particularly in vegetable crop cultivation. Efficient use of available nutrients is more important than other production constraints in vegetable production. Application of organic manures and water soluble fertilizers are such approaches to enhance the nutrient availability. They continuously supply all the essential nutrients throughout the crop period and hence deserve use in vegetable production.

\section{Materials and Methods}

An experiment was conducted during kharif 2013-14 and 2014-15 in farmer's field in Dharwad district situated in the northern transitional zone of Karnataka. The soil of the experimental site was Typic Chromustert and soil reaction was neutral (7.44). The organic carbon content of experimental soil was $6.10 \mathrm{~g}$ $\mathrm{kg}^{-1}$, low in available nitrogen (171 kg ha-1), medium in $\mathrm{P}_{2} \mathrm{O}_{5}\left(25.8 \mathrm{~kg} \mathrm{ha}^{-1}\right)$ and medium in $\mathrm{K}_{2} \mathrm{O}\left(235 \mathrm{~kg} \mathrm{ha}^{-1}\right)$. The experiment consists of four main treatments as organics, $\mathrm{M}_{1^{-}}$ Recommended Package of Practices (RPP)-
\{Recommended Dose of Fertilizers (RDF) + $\left.25 \mathrm{t} \mathrm{ha}^{-1} \mathrm{FYM}\right\}, \mathrm{M}_{2^{-}} 50 \% \mathrm{~N}$ through FYM + $50 \%$ inorganic $\mathrm{N}, \mathrm{M}_{3}-50 \% \mathrm{~N}$ through $\mathrm{VC}+$ $50 \%$ inorganic $\mathrm{N}, \mathrm{M}_{4}-50 \% \mathrm{~N}$ through $\mathrm{FYM}$ and $\mathrm{VC}+50 \%$ inorganic $\mathrm{N}$ and four sub treatments as water soluble fertilizers, $\mathrm{S}_{1^{-}}$ $\mathrm{KNO}_{3} @ 1 \%, \mathrm{~S}_{2}-\mathrm{K}_{2} \mathrm{SO}_{4} @ 1 \%, \mathrm{~S}_{3}-19: 19: 19$ @1\%, $\mathrm{S}_{4}-\mathrm{KNO}_{3}+\mathrm{K}_{2} \mathrm{SO}_{4}+$ 19:19:19 each@ $1 \%$ along with one control (RPP + water spray) with three replications. The design adopted was split plot. The cultivar used for the experiment was Dyvanur with a spacing of $75 \times 75 \mathrm{~cm}$. Foliar sprays were given on 45th DAT (Days After Transplanting) and 90th DAT except for the sub plot treatment $\left(\mathrm{S}_{4}\right)$ which received combined spray of $\mathrm{KNO}_{3}$, $\mathrm{K}_{2} \mathrm{SO}_{4}$, 19:19:19 given at 45th DAT (Days After Transplanting) with 10 days interval for each spray. Total duration of the crop was 140 days.

Nutrient uptake: Plant samples collected for estimation of dry matter accumulation at 75, 105 , and 140 days after transplanting analyzed for iron, zinc, manganese and copper. Micronutrients in the plant samples were determined by the method given by Tandon, 1998. Total uptake of micronutrients was calculated separately by the following formula:

Nutrient uptake $\left(\mathrm{g} \mathrm{ha}^{-1}\right)=$

Nutrient concentration (\%)

100

\section{Results and Discussion}

\section{Capsaicin}

Higher capsaicin content was recorded with the substitution of organic manures compared to control (Table 1). This might be due to higher nitrogen uptake from the organic substituted treatments due to slow and steady supply of nitrogen to plants closely 
synchronizing with capsaicin synthesis in chilli fruits. These results are in conformity with Somimol (2012), who reported that nitrogen content of whole fruit bears significant positive relationship with capsaicin content. Foliar spray of 19:19:19 resulted in higher capsaicin content in fruits. Capsaicin is an alkaloid and is a vanillylamide of isodecyclenic acid. 19:19:19 fertilizer contains 4 per cent $\mathrm{NO}_{3}-\mathrm{N}, 4.50$ per cent $\mathrm{NH}_{4}-\mathrm{N}$ and 10.50 per cent $\mathrm{NH}_{2}-\mathrm{N}$.

The $\mathrm{NH}_{2}$ molecule present in capsaicin structure is responsible for pungency. Foliar spray of 19:19:19 fertilizer resulted in higher nitrogen uptake and the absorbed nitrogen $\left(\mathrm{NH}_{2}-\mathrm{N}\right)$ by the foliage appeared to play a direct role in the formation of amide molecule present in the side chain of capsaicin structure as indicated in biochemical pathway. The present findings are in accordance with the findings of Ananthakrishna and Govindarajan (1975) who reported the synthesis of amide molecule in capsaicin structure as closely related to nitrogen uptake.

Further high nitrogen uptake by plants in the treatment, that received 19:19:19 foliar spray resulted in increased size of plant canopy and leaf. It is reported by Bennet and Kirby (1968) that, a direct relationship exist between leaf area and capsaicin content in fruits, because capsaicin gets synthesized from photosynthates like phenylalanine, valine and leucine which are manufactured in leaves. Control recorded lowest capsaicin content $(0.08 \%)$ on account of water spray and 100 per cent RDN which might not be sufficient for enhancing capsaicin content.

\section{Oleoresin}

Oleoresin is a viscous, semi-solid gel like substance which contains essential oil as well as non-volatile constituents extracted from chillies. Oleoresin permits better and uniform distribution of flavour to food stuffs and it is added to the food in a diluted form. The oleoresin content was significantly higher (Table 1) in organic substituted treatments compared to control (RPP + water spray). The increase in oleoresin content might be attributed to higher uptake of nutrients particularly potassium which was positively and significantly correlated to oleoresin of fruits. These results are in agreement with the findings of Pither and Hall (1990) in tomato and Malawadi (2003) in chilli who have reported higher content of oleoresin with the application of FYM and micronutrients. Santoshkumar and Shashidhara (2006) also obtained higher oleoresin content in chilli fruits with the application of FYM. Thimma (2006) also observed increased oleoresin content from 12.30 to 14.0 per cent with the application of organics viz., poultry manure, vermicompost and FYM as sources of nutrients to chilli.

\section{Micronutrients}

Substitution of 50 per cent nitrogen through vermicompost +50 per cent nitrogen through inorganics noticed higher iron uptake (852.9, 921.6 and $1010.9 \mathrm{~g} \mathrm{ha}^{-1}$ at 75, 105 and 140 DAT respectively), zinc uptake (100.1, 104.9 and $147 \mathrm{~g} \mathrm{ha}^{-1}$ at 75, 105 and 140 DAT respectively), manganese uptake $(274.7,383$ and $408.9 \mathrm{~g} \mathrm{ha}^{-1}$ at 75, 105 and 140 DAT respectively) and copper uptake (48.68, 49.56 and $51.35 \mathrm{~g} \mathrm{ha}^{-1}$ at 75, 105 and 140 DAT respectively) (Table 2 to 5 ).

Lowest uptake was recorded in control treatment. Higher uptake is because of better chelation of native micronutrient cations present in soil with decomposing products of vermicompost. Since vermicompost is in fine powder form, decomposition takes place at faster rate. But in FYM applied plots chelation might take place at slower rate because of coarse nature of FYM. 
Table.1 Capsaicin and Oleoresin content (\%) of sun dried red chilli fruits (Cv. Dyavnur) as influenced by organics and foliar spray of water soluble fertilizers

\begin{tabular}{|c|c|c|c|c|c|c|c|c|c|c|}
\hline \multirow[t]{2}{*}{ Treatments } & \multicolumn{5}{|c|}{ Capsaicin } & \multicolumn{5}{|c|}{ Oleoresin } \\
\hline & $\mathbf{S}_{1}$ & $\mathbf{S}_{2}$ & $\mathbf{S}_{\mathbf{3}}$ & $\mathbf{S}_{4}$ & Mean & $\mathbf{S}_{1}$ & $\mathbf{S}_{2}$ & $\mathbf{S}_{\mathbf{3}}$ & $\mathbf{S}_{4}$ & Mean \\
\hline $\mathbf{M}_{1}$ & 0.11 & 0.10 & 0.13 & 0.12 & 0.11 & 14.83 & 15.58 & 14.07 & 16.29 & 15.19 \\
\hline $\mathbf{M}_{2}$ & 0.10 & 0.10 & 0.14 & 0.14 & 0.12 & 14.92 & 16.12 & 13.97 & 16.32 & 15.33 \\
\hline $\mathbf{M}_{3}$ & 0.11 & 0.10 & 0.14 & 0.14 & 0.12 & 15.45 & 16.48 & 14.28 & 16.60 & 15.70 \\
\hline $\mathbf{M}_{4}$ & 0.11 & 0.11 & 0.13 & 0.13 & 0.12 & 15.54 & 15.80 & 14.63 & 16.19 & 15.54 \\
\hline Mean & 0.11 & 0.10 & 0.14 & 0.13 & & 15.18 & 15.99 & 14.24 & 16.35 & \\
\hline Control & \multicolumn{5}{|c|}{0.08} & \multicolumn{5}{|c|}{13.64} \\
\hline Sources & \multicolumn{2}{|c|}{ S.Em \pm} & \multicolumn{3}{|c|}{$\mathrm{CD}(\mathrm{P}=\mathbf{0 . 0 5})$} & \multicolumn{2}{|c|}{ S.Em \pm} & \multicolumn{3}{|c|}{$\mathrm{CD}(P=0.05)$} \\
\hline $\mathbf{M}$ & \multicolumn{2}{|c|}{0.002} & \multicolumn{3}{|c|}{ NS } & \multicolumn{2}{|c|}{0.36} & \multicolumn{3}{|c|}{ NS } \\
\hline $\mathbf{S}$ & \multicolumn{2}{|c|}{0.002} & \multicolumn{3}{|c|}{0.01} & \multicolumn{2}{|c|}{0.31} & \multicolumn{3}{|c|}{0.90} \\
\hline $\mathbf{M x S}$ & \multicolumn{2}{|c|}{0.004} & \multicolumn{3}{|c|}{ NS } & \multicolumn{2}{|c|}{0.62} & \multicolumn{3}{|c|}{ NS } \\
\hline Control & \multicolumn{2}{|c|}{0.004} & \multicolumn{3}{|c|}{0.01} & \multicolumn{2}{|c|}{0.38} & \multicolumn{3}{|c|}{1.10} \\
\hline
\end{tabular}

\section{Note:}

$\mathrm{M}_{1}-\mathrm{RPP}$
inorganic $\mathrm{N}$

inorganic N

$\mathrm{M}_{3}-50 \% \mathrm{~N}$ through $\mathrm{VC}+50 \%$ inorganic $\mathrm{N}$

$\mathrm{M}_{4}-50 \% \mathrm{~N}$ through FYM and VC $+50 \%$ $\mathrm{S}_{1}-\mathrm{KNO}_{3} @ 1 \%$ 
Table.2 Iron uptake (g ha-1) by plant at different growth stages as influenced by organics and foliar spray of water soluble fertilizers

\begin{tabular}{|c|c|c|c|c|c|c|c|c|c|c|c|c|c|c|c|}
\hline \multirow[t]{2}{*}{ Treatments } & \multicolumn{5}{|c|}{75 DAT } & \multicolumn{5}{|c|}{105 DAT } & \multicolumn{5}{|c|}{140 DAT } \\
\hline & $\mathbf{S}_{1}$ & $\mathbf{S}_{2}$ & $\mathbf{S}_{\mathbf{3}}$ & $\mathbf{S}_{4}$ & Mean & $\mathbf{S}_{1}$ & $\mathbf{S}_{2}$ & $\mathbf{S}_{\mathbf{3}}$ & $\mathbf{S}_{4}$ & Mean & $\mathbf{S}_{1}$ & $\mathbf{S}_{2}$ & $\mathbf{S}_{\mathbf{3}}$ & $\mathbf{S}_{4}$ & Mean \\
\hline$\overline{M_{1}}$ & 665.9 & 630.9 & 689.3 & 691.9 & 669.5 & 729.0 & 711.6 & 742.9 & 753.7 & 734.3 & 803.9 & 781.3 & 811.2 & 827.9 & 806.1 \\
\hline $\mathbf{M}_{2}$ & 733.6 & 711.6 & 759.6 & 773.1 & 744.5 & 793.0 & 781.9 & 797.8 & 824.6 & 799.3 & 888.1 & 878.5 & 899.8 & 923.1 & 897.4 \\
\hline $\mathbf{M}_{3}$ & 837.6 & 820.8 & 868.1 & 885.0 & 852.9 & 911.2 & 873.5 & 938.4 & 963.2 & 921.6 & 1010.4 & 982.8 & 1015.3 & 1035.3 & 1010.9 \\
\hline $\mathbf{M}_{4}$ & 780.5 & 744.2 & 806.7 & 836.5 & 792.0 & 832.0 & 817.0 & 836.8 & 861.6 & 836.8 & 927.5 & 919.9 & 963.2 & 978.8 & 947.3 \\
\hline Mean & 754.4 & 726.9 & 780.9 & 796.6 & & 816.3 & 796.0 & 829.0 & 850.8 & & 907.5 & 890.6 & 922.4 & 941.3 & \\
\hline $\mathbf{M}$ & \multicolumn{2}{|c|}{15.99} & \multicolumn{3}{|c|}{55.35} & \multicolumn{2}{|c|}{7.99} & \multicolumn{3}{|c|}{27.64} & \multicolumn{2}{|c|}{18.19} & \multicolumn{3}{|c|}{62.95} \\
\hline $\mathbf{S}$ & \multicolumn{2}{|c|}{17.69} & \multicolumn{3}{|c|}{ NS } & \multicolumn{2}{|c|}{13.24} & \multicolumn{3}{|c|}{ NS } & \multicolumn{2}{|c|}{13.99} & \multicolumn{3}{|c|}{ NS } \\
\hline $\mathbf{M x S}$ & \multicolumn{2}{|c|}{35.37} & \multirow{2}{*}{\multicolumn{3}{|c|}{ NS }} & \multirow{2}{*}{\multicolumn{2}{|c|}{26.48}} & \multirow{2}{*}{\multicolumn{3}{|c|}{ NS }} & \multicolumn{2}{|c|}{27.97} & \multicolumn{3}{|c|}{ NS } \\
\hline Control & \multicolumn{2}{|c|}{17.24} & & & & & 13.48 & \multicolumn{2}{|r|}{38.84} & & 19 & & & 56.53 & \\
\hline
\end{tabular}

\section{Note:}

$\mathrm{M}_{1}-\mathrm{RPP} \quad \mathrm{M}_{2}-50 \% \mathrm{~N}$ through FYM $+50 \%$ inorganic

inorganic $\mathrm{N}$

$\mathrm{S}_{1}-\mathrm{KNO}_{3} @ 1 \%$

$\mathrm{S}_{2}-\mathrm{K}_{2} \mathrm{SO}_{4} @ 1 \%$

$\mathrm{M}_{3}-50 \% \mathrm{~N}$ through VC $+50 \%$ inorganic $\mathrm{N}_{4}-50 \% \mathrm{~N}$ through $\mathrm{FYM}$ and $\mathrm{VC}+50 \%$

$\mathrm{S}_{3}-19: 19: 19 @ 1 \%$

$\mathrm{S}_{4}-\mathrm{KNO}_{3}+\mathrm{K}_{2} \mathrm{SO}_{4}+19: 19: 19$ each @ 1\%

Control - RPP with water spray, Recommended dose of P and K are common to all the treatments, DAT- Days After Transplanting NS - Non-significant 
Table.3 Zinc uptake (g ha-1) by plant at different growth stages as influenced by organics and foliar spray of water soluble fertilizers

\begin{tabular}{|c|c|c|c|c|c|c|c|c|c|c|c|c|c|c|c|}
\hline \multirow[t]{2}{*}{ Treatments } & \multicolumn{5}{|c|}{75 DAT } & \multicolumn{5}{|c|}{$105 \mathrm{DAT}$} & \multicolumn{5}{|c|}{140 DAT } \\
\hline & $\mathbf{S}_{1}$ & $\mathbf{S}_{2}$ & $\mathbf{S}_{3}$ & $\mathbf{S}_{4}$ & Mean & $\mathbf{S}_{1}$ & $\mathbf{S}_{2}$ & $\mathbf{S}_{3}$ & $\mathbf{S}_{4}$ & Mean & $\mathbf{S}_{1}$ & $\mathbf{S}_{2}$ & $\mathbf{S}_{\mathbf{3}}$ & $\mathbf{S}_{4}$ & Mean \\
\hline $\mathbf{M}_{1}$ & 75.36 & 69.00 & 75.86 & 79.30 & 74.88 & 81.77 & 79.93 & 83.58 & 87.02 & 83.07 & 115.4 & 113.5 & 116.7 & 122.0 & 116.9 \\
\hline$\overline{\mathbf{M}_{2}}$ & 84.66 & 78.87 & 87.43 & 87.11 & 84.52 & 91.25 & 87.37 & 90.19 & 93.95 & 90.69 & 124.1 & 119.0 & 122.3 & 132.2 & 124.4 \\
\hline $\mathbf{M}_{3}$ & 98.14 & 95.23 & 100.8 & 106.1 & 100.1 & 104.6 & 97.44 & 106.5 & 111.0 & 104.9 & 151.4 & 140.7 & 147.3 & 148.6 & 147.0 \\
\hline $\mathbf{M}_{4}$ & 89.80 & 84.56 & 91.09 & 96.89 & 90.58 & 93.75 & 89.51 & 94.27 & 98.61 & 97.34 & 134.6 & 130.6 & 135.8 & 141.9 & 135.7 \\
\hline Mean & 86.99 & 81.91 & 88.80 & 92.35 & & 93.30 & 96.77 & 102.5 & 97.47 & & 131.4 & 125.9 & 130.5 & 136.2 & \\
\hline Control & \multicolumn{5}{|c|}{65.04} & \multicolumn{5}{|c|}{70.73} & \multicolumn{5}{|c|}{98.33} \\
\hline Sources & \multicolumn{2}{|c|}{ S.Em \pm} & \multicolumn{3}{|c|}{$\mathrm{CD}(\mathrm{P}=\mathbf{0 . 0 5})$} & \multicolumn{2}{|c|}{ S.Em \pm} & \multicolumn{3}{|c|}{$\mathrm{CD}(\mathrm{P}=0.05)$} & \multicolumn{2}{|c|}{ S.Em \pm} & \multicolumn{3}{|c|}{$\mathrm{CD}(\mathrm{P}=\mathbf{0 . 0 5})$} \\
\hline $\mathbf{M}$ & \multicolumn{2}{|c|}{2.17} & \multicolumn{3}{|c|}{7.51} & \multicolumn{2}{|c|}{1.90} & \multicolumn{3}{|c|}{6.56} & \multicolumn{2}{|c|}{1.06} & \multicolumn{3}{|c|}{3.67} \\
\hline $\mathbf{S}$ & \multicolumn{2}{|c|}{2.51} & \multicolumn{3}{|c|}{ NS } & \multicolumn{2}{|c|}{2.15} & \multicolumn{3}{|c|}{ NS } & \multicolumn{2}{|c|}{2.44} & \multicolumn{3}{|c|}{ NS } \\
\hline $\mathbf{M x S}$ & \multicolumn{2}{|c|}{5.03} & \multicolumn{3}{|c|}{ NS } & \multicolumn{2}{|c|}{4.30} & \multicolumn{3}{|c|}{ NS } & \multicolumn{2}{|c|}{4.88} & \multicolumn{3}{|c|}{ NS } \\
\hline Control & \multicolumn{2}{|c|}{2.50} & \multicolumn{3}{|c|}{7.19} & \multicolumn{2}{|c|}{3.03} & \multicolumn{3}{|c|}{8.71} & & & & 8.79 & \\
\hline
\end{tabular}

Note:

$\mathrm{M}_{1}-\mathrm{RPP} \quad \mathrm{M}_{2}-50 \% \mathrm{~N}$ through $\mathrm{FYM}+50 \%$ inorganic $\mathrm{N}$

inorganic $\mathrm{N}$

$\mathrm{S}_{1}-\mathrm{KNO}_{3} @ 1 \%$

$\mathrm{S}_{2}-\mathrm{K}_{2} \mathrm{SO}_{4} @ 1 \%$

$\mathrm{M}_{3}-50 \% \mathrm{~N}$ through $\mathrm{VC}+50 \%$ inorganic $\mathrm{N} \mathrm{M}_{4}-50 \% \mathrm{~N}$ through $\mathrm{FYM}$ and $\mathrm{VC}+50 \%$

$\mathrm{S}_{3}-19: 19: 19 @ 1 \%$

$\mathrm{S}_{4}-\mathrm{KNO}_{3}+\mathrm{K}_{2} \mathrm{SO}_{4}+19: 19: 19$ each @ 1\%

Control - RPP with water spray, Recommended dose of P and K are common to all the treatments, DAT- Days After Transplanting NS - Non-significant 
Table.4 Manganese uptake (g ha-1) by plant at different growth stages as influenced by organics and foliar spray of water soluble fertilizers

\begin{tabular}{|c|c|c|c|c|c|c|c|c|c|c|c|c|c|c|c|}
\hline \multirow[t]{2}{*}{ Treatments } & \multicolumn{5}{|c|}{75 DAT } & \multicolumn{5}{|c|}{105 DAT } & \multicolumn{5}{|c|}{140 DAT } \\
\hline & $\mathbf{S}_{1}$ & $\mathbf{S}_{2}$ & $\mathbf{S}_{\mathbf{3}}$ & $\mathbf{S}_{4}$ & Mean & $\mathbf{S}_{1}$ & $\mathbf{S}_{\mathbf{2}}$ & $\mathbf{S}_{\mathbf{3}}$ & $\mathbf{S}_{4}$ & Mean & $\mathbf{S}_{1}$ & $\mathbf{S}_{\mathbf{2}}$ & $\mathbf{S}_{\mathbf{3}}$ & $\mathbf{S}_{4}$ & Mean \\
\hline $\mathbf{M}_{1}$ & 212.4 & 195.4 & 216.0 & 215.0 & 209.7 & 311.3 & 301.4 & 308.4 & 318.8 & 310.0 & 352.8 & 343.3 & 349.4 & 353.6 & 349.8 \\
\hline$\overline{\mathbf{M}_{2}}$ & 232.6 & 223.7 & 236.2 & 246.9 & 234.8 & 333.9 & 328.2 & 339.9 & 346.2 & 337.1 & 361.7 & 362.6 & 366.4 & 377.4 & 367.0 \\
\hline $\mathbf{M}_{3}$ & 272.6 & 263.5 & 276.5 & 286.1 & 274.7 & 381.3 & 362.0 & 386.5 & 402.3 & 383.0 & 411.4 & 397.1 & 406.9 & 420.3 & 408.9 \\
\hline $\mathbf{M}_{4}$ & 250.9 & 236.3 & 255.7 & 275.0 & 254.5 & 352.8 & 344.7 & 361.1 & 369.6 & 357.0 & 382.8 & 381.1 & 398.5 & 399.1 & 390.4 \\
\hline Mean & 242.1 & 229.7 & 246.1 & 255.8 & & 344.8 & 334.1 & 349.0 & 359.2 & & 377.2 & 371.0 & 380.3 & 387.6 & \\
\hline Control & \multicolumn{5}{|c|}{163.7} & \multicolumn{5}{|c|}{ 251.0 } & \multicolumn{5}{|c|}{303.5} \\
\hline Sources & \multicolumn{2}{|c|}{ S.Em \pm} & \multicolumn{3}{|c|}{$\mathrm{CD}(\mathrm{P}=0.05)$} & \multicolumn{2}{|c|}{ S.Em \pm} & \multicolumn{3}{|c|}{$\mathrm{CD}(\mathrm{P}=0.05)$} & \multicolumn{2}{|c|}{ S.Em \pm} & \multicolumn{3}{|c|}{$\mathrm{CD}(\mathrm{P}=\mathbf{0 . 0 5})$} \\
\hline $\mathbf{M}$ & \multicolumn{2}{|c|}{4.03} & \multicolumn{3}{|c|}{13.96} & \multicolumn{2}{|c|}{5.11} & \multicolumn{3}{|c|}{17.67} & \multicolumn{2}{|c|}{3.28} & \multicolumn{3}{|c|}{11.34} \\
\hline $\mathbf{S}$ & \multicolumn{2}{|c|}{6.21} & \multicolumn{3}{|c|}{ NS } & \multicolumn{2}{|c|}{6.02} & \multicolumn{3}{|c|}{ NS } & \multicolumn{2}{|c|}{4.14} & \multicolumn{3}{|c|}{ NS } \\
\hline MxS & \multicolumn{2}{|c|}{12.43} & \multicolumn{3}{|c|}{ NS } & \multicolumn{2}{|c|}{12.05} & \multicolumn{3}{|c|}{ NS } & \multicolumn{2}{|c|}{8.27} & \multicolumn{3}{|c|}{ NS } \\
\hline Control & \multicolumn{2}{|c|}{5.65} & \multicolumn{3}{|c|}{16.28} & \multicolumn{2}{|c|}{7.51} & \multicolumn{3}{|c|}{21.64} & & & & 16.35 & \\
\hline
\end{tabular}

Note:

$\mathrm{M}_{1}-\mathrm{RPP}$
inorganic $\mathrm{N}$

inorganic $\mathrm{N}$

$\mathrm{M}_{3}-50 \% \mathrm{~N}$ through $\mathrm{VC}+50 \%$ inorganic $\mathrm{N} \mathrm{M}_{4}-50 \% \mathrm{~N}$ through FYM and $\mathrm{VC}+50 \%$ $\mathrm{S}_{1}-\mathrm{KNO}_{3} @ 1 \%$

$\mathrm{S}_{2}-\mathrm{K}_{2} \mathrm{SO}_{4} @ 1 \%$

$\mathrm{S}_{3}-19: 19: 19 @ 1 \%$

$\mathrm{S}_{4}-\mathrm{KNO}_{3}+\mathrm{K}_{2} \mathrm{SO}_{4}+19: 19: 19$ each @ 1\%

Control - RPP with water spray, Recommended dose of P and K are common to all the treatments, DAT- Days After Transplanting NS - Non-significant 
Table.5 Copper uptake (g ha-1) by plant at different growth stages as influenced by organics and foliar spray of water soluble fertilizers

\begin{tabular}{|c|c|c|c|c|c|c|c|c|c|c|c|c|c|c|c|}
\hline \multirow[t]{2}{*}{ Treatments } & \multicolumn{5}{|c|}{75 DAT } & \multicolumn{5}{|c|}{105 DAT } & \multicolumn{5}{|c|}{140 DAT } \\
\hline & $\mathbf{S}_{1}$ & $\mathbf{S}_{2}$ & $\mathbf{S}_{\mathbf{3}}$ & $\mathbf{S}_{4}$ & Mean & $\mathbf{S}_{1}$ & $\mathbf{S}_{\mathbf{2}}$ & $\mathbf{S}_{\mathbf{3}}$ & $\mathbf{S}_{4}$ & Mean & $\mathbf{S}_{1}$ & $\mathbf{S}_{2}$ & $\mathbf{S}_{\mathbf{3}}$ & $\mathbf{S}_{4}$ & Mean \\
\hline $\mathbf{M}_{1}$ & 34.54 & 31.68 & 33.42 & 36.02 & 33.92 & 38.69 & 37.92 & 40.08 & 42.08 & 39.70 & 41.09 & 40.97 & 41.57 & 43.45 & 41.77 \\
\hline$\overline{\mathbf{M}_{2}}$ & 38.45 & 34.35 & 36.79 & 40.70 & 37.57 & 42.37 & 41.18 & 43.30 & 46.93 & 43.44 & 43.59 & 42.34 & 45.02 & 48.19 & 44.78 \\
\hline $\mathbf{M}_{3}$ & 47.49 & 46.94 & 48.67 & 51.63 & 48.68 & 49.66 & 46.31 & 50.60 & 51.67 & 49.56 & 51.47 & 48.69 & 52.08 & 53.18 & 51.35 \\
\hline $\mathbf{M}_{4}$ & 43.05 & 41.88 & 43.10 & 45.68 & 43.43 & 46.58 & 44.75 & 46.45 & 48.22 & 46.50 & 48.32 & 46.23 & 49.27 & 50.13 & 48.49 \\
\hline Mean & 40.88 & 38.71 & 40.50 & 43.51 & & 44.33 & 42.54 & 45.11 & 47.22 & & 46.12 & 44.56 & 46.98 & 48.74 & \\
\hline Control & \multicolumn{5}{|c|}{28.60} & \multicolumn{5}{|c|}{34.12} & \multicolumn{5}{|c|}{35.72} \\
\hline Sources & \multicolumn{2}{|c|}{ S.Em \pm} & \multicolumn{3}{|c|}{$\mathrm{CD}(\mathrm{P}=0.05)$} & \multicolumn{2}{|c|}{ S.Em \pm} & \multicolumn{3}{|c|}{$\mathrm{CD}(\mathrm{P}=0.05)$} & \multicolumn{2}{|c|}{ S.Em \pm} & \multicolumn{3}{|c|}{$\mathrm{CD}(\mathrm{P}=\mathbf{0 . 0 5})$} \\
\hline $\mathbf{M}$ & \multicolumn{2}{|c|}{1.08} & \multicolumn{3}{|c|}{3.75} & \multicolumn{2}{|c|}{0.83} & \multicolumn{3}{|c|}{2.87} & \multicolumn{2}{|c|}{$1.00^{-}$} & \multicolumn{3}{|c|}{3.47} \\
\hline $\mathbf{S}$ & \multicolumn{2}{|c|}{1.15} & \multicolumn{3}{|c|}{ NS } & \multicolumn{2}{|c|}{1.14} & \multicolumn{3}{|c|}{ NS } & \multicolumn{2}{|c|}{1.04} & \multicolumn{3}{|c|}{ NS } \\
\hline MxS & \multicolumn{2}{|c|}{2.30} & \multicolumn{3}{|c|}{ NS } & \multicolumn{2}{|c|}{2.28} & \multicolumn{3}{|c|}{ NS } & \multicolumn{2}{|c|}{2.08} & \multicolumn{3}{|c|}{ NS } \\
\hline Control & \multicolumn{2}{|c|}{1.47} & \multicolumn{3}{|c|}{4.24} & \multicolumn{2}{|c|}{1.51} & \multicolumn{3}{|c|}{4.35} & & & & 4.95 & \\
\hline
\end{tabular}

\section{Note:}

$\mathrm{M}_{1}-\mathrm{RPP} \quad \mathrm{M}_{2}-50 \% \mathrm{~N}$ through $\mathrm{FYM}+50 \%$ inorganic $\mathrm{N}$

inorganic $\mathrm{N}$

$\mathrm{M}_{3}-50 \% \mathrm{~N}$ through $\mathrm{VC}+50 \%$ inorganic $\mathrm{N} \mathrm{M}_{4}-50 \% \mathrm{~N}$ through $\mathrm{FYM}$ and $\mathrm{VC}+50 \%$ $\mathrm{S}_{1}-\mathrm{KNO}_{3} @ 1 \%$

Control - RPP with water spray, Recommended dose of P and K are common to all the treatments, DAT- Days After Transplanting NS - Non-significant 
Further organics substituted treatments recorded higher micronutrients uptake than RPP which was attributed to decomposition of organic manures and the decomposing products of organic manures form chelates with native micronutrients enhancing their availability and uptake by chilli crop. The extent of micronutrients uptake was higher at 50 per cent substitution of nitrogen through organics. This might be due to the faster decomposition of organic manures as a result of narrowed $\mathrm{C}: \mathrm{N}$ ratio with the combined application of both organic and inorganic sources of nutrients. This increased the availability of cationic micronutrient content in soil solution that led to increased uptake by plants (Poongothi and Mathan, 2000).

Application of organic manures and foliar spray of different water soluble fertilizers has enhanced capsaicin, oleoresin and nutrient uptake of chilli. Combined foliar spray of $\mathrm{KNO}_{3}$ $+\mathrm{K}_{2} \mathrm{SO}_{4}+19: 19: 19$ each at one per cent along substitution of 50 per cent $\mathrm{N}$ through $\mathrm{VC}+50$ per cent inorganic $\mathrm{N}$ has shown highest uptake but economics of using this is a concern.

\section{References}

Ananthakrishna, S. M. and Govindarajan, V. S., 1975, Evaluation of spices and oleoresins and estimation of pungent principles of pepper (Piper nigrum L.). J. Food Sci. Tech., 12: 253-256.

Bennet, D. J. and Kirby, G. W., 1968, Constitution and biosynthesis of capsaicin. J. Chemical Soc. Colarado, p. 442.

FAO (2007) Production Yearbook. Food and Agriculture Organization Rome.

Lester GE, John LJ, Donald JM (2010) Impact of potassium nutrition on food quality of fruits and vegetables. Better Crops 94: 18-21.

Malawadi, M. N., 2003, Effect of secondary and micronutrients on yield and quality of chilli (Capsicum annuum L.). M.Sc. (Agri.) Thesis, Univ. Agric. Sci., Dharwad (India).

Pither, R. and Hall, M. N., 1990, Analytical survey of the nutritional composition of organically grown fruits and vegetables. Technical Memorandum, 597, MAFF project-4350, Campdon Food and Drink Research Association.

Poongothi, S. and Mathan, K. K., 2000, Effect of organic manures and copper application on the uptake micronutrients by maize. Agric. Sci. Digest, 20: 215-218.

Prasad BC, Kumar V, Gururaj HB, Parimalan R, Giridhar P (2006) Characterization of capsaicin synthase and identification of pungency factor capsaicin in pepper (Capsicum Spp.). P Natl Acad Sci 103: 13315-13320.

Santoshkumar, K. and Shashidhara, G. B., 2006, Integrated nutrient management in chilli genotypes. Karnataka J. Agric. Sci., 19(3): 506-512.

Somimol, P. V., 2012, Effect of foliar feeding of 19:19:19 and potassium nitrate water soluble fertilizers on yield and quality of Byadgi chillies in a Vertisol. M. Sc. (Agri.) Thesis, Univ. Agric. Sci., Dharwad (India).

Tandon, H. L. S., 1998, Methods of Analysis of Soils, Plants, Water and Fertilizers. Fertilizer Development and Consultation Organization, 31: 9-16.

Thimma, N. M., 2006, Studies on the effect of organic manures on growth, yield and quality of chilli (Capsicum annuum L.) under northern transition zone of Karnataka. M.Sc. (Agri.) Thesis, Univ. Agric. Sci., Dharwad (India).

\section{How to cite this article:}

Thulasiramireddy, T., B.I. Bidari, P.L. Patil and Manjunatha, M.V. 2018. Studies on Nitrogen Substitution through Organics and Foliar Application of Water Soluble Fertilizers on Chemical Parameters and Micro Nutrient Uptake by Chilli (Capsicum annuum L.) In a Vertisol. Int.J.Curr.Microbiol.App.Sci. 7(12): 3023-3031. doi: https://doi.org/10.20546/ijcmas.2018.712.347 